Revue pluridisciplinaire d'études médiévales

\title{
Le turban fait-il l'oriental ? Les chrétiens de la ceinture dans les récits de pèlerinage occidentaux (XIII ${ }^{\mathrm{e}}-\mathrm{XIV}^{\mathrm{e}}$ siècles)
}

\section{Camille Rouxpetel}

\section{CpenEdition}

\section{Journals}

Édition électronique

URL : http://journals.openedition.org/questes/91

DOI : 10.4000/questes.91

ISSN : 2109-9472

\section{Éditeur}

Les Amis de Questes

\section{Édition imprimée}

Date de publication : 15 avril 2013

Pagination : 23-44

ISSN : 2102-7188

\section{Référence électronique}

Camille Rouxpetel, « Le turban fait-il l'oriental ? Les chrétiens de la ceinture dans les récits de pèlerinage occidentaux (XIII -XIVe siècles) », Questes [En ligne], 25 | 2013, mis en ligne le 01 janvier 2014, consulté le 01 mai 2019. URL : http://journals.openedition.org/questes/91 ; DOI : 10.4000/ questes. 91 


\title{
Le turban fait-il l'oriental ? Les chrétiens de la ceinture dans les récits de pèlerinage occidentaux $\left(\mathrm{XIII}^{\mathrm{e}}-\mathrm{XIV}^{\mathrm{e}}\right.$ siècles)
}

\author{
Camille ROUAPETEL \\ Université Paris-Sorbonne (Paris IV)
}

Roland Barthes, dans un article des Annales E.S.C. de 1957, établit une parenté entre langage et vêtement, entre système linguistique et système vestimentaire, tous deux conçus comme des systèmes de signes ${ }^{1}$. À cet égard, le sociologue pose une différence entre le vêtement et le costume, plus intéressant à ses yeux car il marque l'appartenance à un groupe. De leur côté, la majorité des récits de pèlerinage qui mentionnent les vêtements portés par les chrétiens d'Orient les considèrent comme un signe à interpréter. Les pèlerins s'intéressent peu aux vêtements liturgiques ou monastiques des chrétiens d'Orient, à l'exception notable de Burchard de Mont Sion dans le contexte de son séjour en Cilicie dans les années 1274-1283. Ils assistent pourtant à de nombreuses messes célébrées selon les rites des différentes communautés chrétiennes orientales, ainsi qu'ils le précisent systématiquement. Ils décrivent alors la liturgie, les chants, les objets utilisés, les différences dans les modalités de la célébration eucharistique. Peut-être les vêtements sont-ils si semblables à ceux portés en Occident que les pèlerins ne voient pas l'intérêt de les mentionner particulièrement. De fait, l'essentiel des remarques qui concernent les vêtements portés par les chrétiens d'Orient se rapportent à des vêtements ou à des éléments de vêtements qui sortent de l'ordinaire - ceinture et turban - et renvoient ceux qui les revêtent à une identité ou une altérité

Roland BARTHES, "Histoire et sociologie du vêtement. Quelques observations méthodologiques », Annales E.S.C., 3 (juillet-septembre 1957), p. 430-441. 
orientale. Aussi ces remarques sont-elles sans doute le reflet de la curiositas de ces pèlerins médiévaux, qui sont également des voyageurs, voire le signe d'un goût pour l'« exotisme », goût qui s'accentue à la fin du Moyen Âge, et particulièrement à partir du XIV siècle, quand les Latins s'intéressent de plus en plus à leur environnement géographique et humain réel, en décrivant par exemple la diversité des cultures arbustives et légumières ou les marchandises de toutes sortes présentes sur les étals des souks égyptiens ${ }^{2}$. Posant la question de la présence de l'exotisme dans les textes médiévaux, Emmanuèle Baumgartner relève trois traits qui le caractérisent. Il se développe d'abord dans le contexte des récits de voyage dans des pays étranges, donc différents. Il résulte ensuite d'« une certaine attitude mentale d'ouverture vers l'autre ». Enfin, l'espace est évoqué « non pas pour sa dimension mythique ou symbolique, mais dans la mesure où il peut procurer le contact avec des objets ou des êtres nouveaux, qui sont précisément appréciés pour leur nouveauté et leur différence $»^{3}$. Il convient dès lors de s'interroger sur la signification que revêt le vêtement dans le processus d'identification et de caractérisation des chrétiens d'Orient par les pèlerins occidentaux, entre une altérité façonnée par les représentations latines du monachisme oriental et une altérité résultant de l'observation de ce qui sort de l'ordinaire et de l'univers familier des Occidentaux.

\footnotetext{
2 Sur les rapports entretenus par les historiens et les sociologues avec la notion d' " exotisme », voir la mise au point historiographique d'Anaïs FLÉCHET, « L'exotisme comme objet d'histoire », Hypothèses 2007. Travaux de l'École doctorale d'histoire de l'Université Paris I Panthéon-Sorbonne, Paris, Publications de la Sorbonne, 11, 2008, p. $15-26$.

${ }^{3}$ Emmanuèle BAUMGARTNER, «L'exotisme à rebours de la Chanson d'Antioche », dans L'Exotisme dans la poésie épique française, In memoriam Klára Csürös, Actes du colloque international du 26 au 28 octobre 2000, Anikó KALMÁR (dir.), Paris, Budapest/Turin, L'Harmattan, 2003, p. 13-28, citations p. 13.
} 


\section{Le vêtement: signe d'appartenance à une communauté de croyances et de représentations.}

Burchard de Mont Sion décrit les vêtements portés par le patriarche des Arméniens en insistant sur leur grossièreté, symbole de son ascétisme et de sa piété. Il oppose en effet les richesses temporelles que possède le catholicos en vertu de sa fonction (« infinitarum diuiciarum ») à la pauvreté de son vêtement, également caractéristique de sa fonction ecclésiastique («pallium uile»), et à son comportement tout entier tourné vers la piété («Et erat in uictu et uestitu et omni conuersatione sua tanti exempli, quod similem non uidi $»)^{4}$. Cette description s'explique par l'image du monachisme oriental qui prévaut alors en Occident. Cette dernière est fondée sur l'expérience directe issue de rencontres avec des moines grecs et arméniens et sur des textes qui appartiennent à la culture savante et religieuse. Les écrits hagiographiques et patristiques valorisent en effet le monachisme oriental, et particulièrement le monachisme égyptien issu des Pères du désert, pour son ascétisme et son renoncement. Les Ascetica de saint Basile et les diverses Vitce sanctorum Patrum sont lus en Occident comme une source d'édification ${ }^{5}$. Saint Benoît lui-même recommande cette

\footnotetext{
4 « Des richesses en nombre infini »; « un manteau vil »; « Et il y avait, dans sa façon de se nourrir et de s'habiller et dans tout son comportement, tant d'exemplarité, que je n'ai rien vu de semblable », BuRChaRd DE MOnT Sion, Descriptio Terrce Sanctce, dans Peregrinatores Medii Aevi quatuor: Burchardus de Monte Sion, Ricoldus de Monte Crucis, Odoricus de Foro Julii, Wilbrandus de Oldenborg, Johann Christian Moritz LAURENT (éd.), Leipzig, 1864, p.91-92 (nous traduisons). Pour une analyse plus détaillée de ce passage, nous nous permettons de renvoyer à l'article publié dans le bulletin n²4 de Questes, qui porte sur l'identité (Camille ROUXPETEL, "Questionner l'identité d'un chrétien occidental, pèlerin en Terre sainte, face à la diversité des chrétiens d'Orient. L'approche de Burchard de Mont Sion ", dans L'Identité, Cécile Becchia, Diane Chamboduc De Saint Pulgent, Fanny Oudin (dir.), Questes, 24, 2012, p. 48-65, en particulier p. 52-54).

${ }^{5}$ Voir Chiara FARAGgiana DI SARZANA, « Gli insegnamenti dei Padri del deserto nella Roma altomedievale (secc. V-IX) : vie e modi di diffusione ", dans Roma tra Oriente e Occidente, Spolète, Centro italiano di studi sull'alto mediœvo, "Settimane di studio del Centro italiano di studi sull'alto mediœvo », 2002, I, p. 587-602. Sur la fascination exercée par l'ancien monachisme oriental, voir Gregorio PENCO, «Il ricordo
} 
pratique à la fin de sa règle ${ }^{6}$. Par ailleurs, les pèlerinages de moines grecs et arméniens en Occident et l'implantation de monastères grecs et arméniens en Italie favorisent les rencontres avec les Latins ${ }^{7}$. Ainsi, au début du $\mathrm{XIII}^{\mathrm{e}}$ siècle, Willebrand d'Oldenburg se rend en Arménie pour remplir au nom d'Othon IV une mission diplomatique, qui fait suite à la création récente de liens vassaliques entre la royauté cilicienne et le Saint-Empire ${ }^{8}$. Il souligne la piété des Arméniens, entrés depuis 1184 en communion avec l'Église romaine'.

Enfin, l'utilisation du vêtement pour symboliser une vertu chrétienne est courante pour un Latin du XIII ${ }^{\mathrm{e}}$ siècle, comme en témoigne la

dell'ascetismo orientale nella tradizione monastica del medioœvo », dans Studi medievali, IV (1963), p. 571-587, réimpr. dans Gregorio PENCO, Medioevo monastico, Rome, Centro Studi San Anselmo, « Studia Anselmiana », 1988, p. 515-535.

${ }^{6}$ « Necnon et Collationes Patrum et Instituta et Vitas eorum, sed et Regula sancti Patris nostri Basilii » («Et aussi les recueils des Pères, leurs préceptes et leurs vies, mais surtout la Règle de notre saint Père Basile », La Règle de Saint Benoît, Jean NeufVILle (éd.), Adalbert DE VoGÜÉ (trad.), Paris, Éditions du Cerf, « Sources chrétiennes », 1972, II, p. 675. Nous traduisons).

${ }^{7}$ Voir Patricia M. MacNulty et Bernard Hamilton, « "Orientale lumen” et "Magistra Latinitas" : Greek influences on Western Monasticism (900-1100) », dans Le Millénaire du Mont Athos, 963-1963, I, Venise-Chevetogne, Éditions de Chevetogne/Fondazione Giorgio Cini, 1963, p. 181-216, réimpr. dans Bernard Hamilton, Monastic Reform, Catharism and the Crusades (900-1300), Londres, Variorum reprints, "Collected Studies Series », 97, 1979 ; Levon Boghos ZEKIYAN, « Le coloni armene del Medio Evo in Italia e la relazioni culturali italo-armene (Materiale per la Storia degli Armeni in Italia) », dans Atti del primo symposio internazionale di arte armena, Giulio IENI et Levon Boghos ZeKIYAn (dir.), Saint Lazare/Venise, 1978, p. 803-946.

${ }^{8}$ Willebrand D’Oldenburg, Descriptio Terree Sanctee, dans Peregrinatores Medii Aevi quatuor ..., éd. cit., p. 174-180.

${ }^{9}$ En 1184 le patriarche Grégoire Dgha envoie un évêque arménien, porteur d'une profession de foi, auprès du pape Lucius III. La profession de foi a été égarée, mais la réponse du pape a été conservée dans la traduction arménienne réalisée par Nersès de Lampron. Le pape adresse au catholicos une mitre, le pallium, l'anneau, symboles de la reconnaissance de sa juridiction, et une copie du Rituale Romanum, traduit par Nersès. L'envoi de la profession de foi est interprété par Lucius III comme un acte de soumission au siège romain et l'envoi du pallium comme la reconnaissance de la communion de l'Église arménienne avec Rome. Quant à la copie du Rituale Romanum elle est destinée à permettre aux Arméniens d'accorder leurs usages avec la coutume romaine. L'union se manifeste d'abord par un ordre de Lucius III au clergé arménien du diocèse de Valania, qui ne dispose d'aucun évêque propre, de se placer sous l'obédience de l'évêque latin. 
transformation en topos hagiographique, dès le $\mathrm{X}^{\mathrm{e}}$ siècle, du geste de saint Martin partageant son manteau aux portes d'Amiens. Le geste de se dépouiller de son manteau pour autrui devient un symbole qui participe à l'institutionnalisation d'un nouveau modèle chrétien de comportement, dont les ordres mendiants sont en partie les héritiers. Ainsi, au XIII ${ }^{\mathrm{e}}$ siècle, la charité n'est plus représentée par l'aumône faite aux pauvres, mais par la remise d'un vêtement, geste qui signifie l'affranchissement à l'égard d'un signe extérieur de différenciation entre potentes et pauperes. La description de Burchard, si elle se fonde sur des éléments concrets et tangibles - les vêtements du catholicos - est donc également une formalisation de l'idéal spirituel et du mode de vie évangélique choisis par les dominicains et partagés par le clergé arménien, ce qui met en exergue l'unité de la chrétienté.

Cette position se retrouve chez Riccold de Monte Croce, un dominicain des environs de Florence qui part en mission en Orient à la fin du XIII ${ }^{\mathrm{e}}$ siècle. Il débarque à Saint-Jean d'Acre en 1288, au moment où Burchard quitte la Terre sainte, et rentre en Italie une dizaine d'années plus tard. Dans le chapitre qu'il consacre aux nestoriens dans son liber peregrinationis, il les décrit en ces termes :

In habitu siue in uestitu tam religiosi quam episcopi eorum et superiores magnam paupertatem et austeritatem et honestatem et humilitatem ostendunt. In quadragesima tam Nestorini quam Iacobini omnes tam religiosi quam seculares nullo modo comederent pisces nec biberent uinum ${ }^{10}$.

\footnotetext{
${ }^{10}$ «Dans leurs manières et (ou) leur vêture, aussi bien leurs religieux que leurs évêques et leurs prélats se montrent plein de pauvreté, d'austérité, d'honnêteté et d'humilité. À quadragésime, nestoriens et jacobins, religieux comme séculiers, aucun d'eux ne mangerait de poisson ni ne boirait de vin » (RICCOLD DE MONTE CROCE, Pérégrination en Terre Sainte et au Proche Orient, Texte latin et traduction; Lettres sur la chute de Saint-Jean d'Acre, René KAPPLER (éd. et trad.), Paris, Champion, «Textes et traductions des classiques français du Moyen Âge », 1997, p. 134 et 135 pour la traduction).
} 
Dans cet exemple le vêtement revêt à nouveau une signification morale, il révèle (ostendunt) les vertus des prélats nestoriens, conformes à l'idéal apostolique du missionnaire dominicain. Le costume, associé au jeûne et au comportement, fait montre de l'intériorité de la personne, s'inscrit dans la relation dialectique entre foris et intus qui sous-tend la transition de l'intérieur vers l'extérieur de l'être en lien avec l'ambivalence de l'homme telle qu'elle est définie dans les Etymologice d'Isidore de Séville : «Duplex est autem homo, interior et exterior. Interior homo, anima, exterior homo, corpus ${ }^{11}$. La concordance ou l'opposition entre foris et intus contribue alors à fixer le jugement porté par les Latins sur leurs coreligionnaires orientaux, l'habitus pouvant soit révéler l'intériorité, soit la dissimuler, la dissimulation et l'hypocrisie étant reconnues comme des attributs de l'hérésie.

\section{Le vêtement, un marqueur ethnique?}

Dans sa caractérisation des Syriens, c'est-à-dire des chrétiens nés en Terre sainte, Burchard de Mont Sion signale la pauvreté de leur habillement, mais dans une perspective différente de la description du catholicos:

Preter hos sunt ibi Syriani sive Syri. Hij totam repleverunt terram. Christiani tamen sunt, sed latinis nullum fidem servant. Misere vestiuntur et induuntur, parci sunt, eleemosynam non dant. Inter Sarracenos habitant, et ut plurimum eorum officiis funguntur. In habitu concordant

\footnotetext{
11 «L'homme est donc double, intérieur et extérieur. L'homme intérieur, c'est l'âme, l'homme extérieur, le corps » (ISIDORE DE SÉVILLE, Etymologice, dans Patrologice cursus completus ... patres ecclesice latince, Jacques-Paul Migne (éd.), Petit-Montrouge, Paris, 1844-1864, 223 vol. et un supplément en 5 vol., vol. LXXXVII, col. 398, 685. Nous traduisons).
} 
cum Sarracenis, nisi quod tantum per cingulum laneum discerneuntur ${ }^{12}$.

Le vêtement est un indice qui permet à Burchard d'affirmer que ces chrétiens sont pauvres, état qu'il juxtapose à leur parcimonie et au fait qu'ils ne donnent pas d'aumône. Il se situe donc dans une approche rationnelle des chrétiens syriens, qui se fonde sur l'observation empirique de signes visibles et objectifs. Ce passage s'insère toutefois entre sa remarque sur leur absence de fidélité envers les Latins malgré leur foi chrétienne commune ( latinis nullum fidem servant»), et la question des rapports entre les Sarrasins et les Syriens. Burchard lie donc la distance des Syriens vis-à-vis des Latins à leur proximité géographique et quotidienne avec les Sarrasins. Dans ce passage, les Latins et les Syriens, tout en étant proches religieusement, se distinguent ethniquement, ne serait-ce que par l'emploi d'ethnonymes pour les désigner. En revanche, les Syriens vivent parmi les Sarrasins, dont ils semblent se rapprocher ethniquement. Ils sont unis par la terre qu'ils habitent ensemble. Dans ce contexte il semble donc que le vêtement soit un élément de l'identification ethnique, au même titre que le comportement, la manière de vivre: Sarrasins et Syriens vivent ensemble (inter) et sont liés par des relations réglementées par l'échange, les Syriens étant au service des Sarrasins. La description de Burchard est de l'ordre du constat. Il est néanmoins possible de discerner un soupçon d'acidité dans son insistance sur la misère des Syriens et sur leur soumission aux Sarrasins, si on la met en perspective avec la mention de leur absence de fidélité envers les Latins, alors qu'ils partagent la même

\footnotetext{
12 «En plus de ceux-ci [les Sarrasins], il y a les Syriani ou Syri. Ils peuplent toute cette terre. Ils sont certes chrétiens, mais ils ne témoignent aucune fidélité aux Latins. Ils sont vêtus et revêtus misérablement, ils sont économes et ne donnent pas d'aumône. Ils habitent parmi les Sarrasins, vu que la plupart d'entre eux sont à leur service. Ils s'habillent comme les Sarrasins, à ceci près qu'on les distingue par une ceinture de laine » (Burchard DE Mont Sion, Descriptio Terre Sanctce, dans Peregrinatores Medii Aevi quatuor..., éd. cit., p. 89. Nous traduisons).
} 
foi. Tout son développement découle en effet de cette remarque liminaire et en constitue en quelque sorte l'explicitation et la justification. Les Syriens sont pauvres et vivent au milieu des Sarrasins, avec lesquels ils entretiennent des relations fondées sur la dépendance - ils sont à leur service - et sur la hiérarchie - ils sont contraints à porter une ceinture discriminante - ce qui explique, ou du moins explicite, ce que Burchard entend par «latinis nullum fidem servant»: fidem est à comprendre ici dans un triple sens social, économique et politique. Le vêtement et le comportement des Syriens les intègre au groupe ethnique des Sarrasins, avec lesquels ils ont des échanges économiques et contre lesquels ils ne concluent aucune alliance avec les Francs. De même que Burchard utilise le vêtement pour définir la situation des Syriens et les rapprocher ainsi des Sarrasins, de même Jacques de Vérone, frère augustin dont le pèlerinage en Terre sainte date de la seconde moitié de l'année 1335, utilise le vêtement pour caractériser les Géorgiens :

quia Georgiani sunt induti ad modum Saracenorum preterquam in capite. Quia Saraceni portant melmam albam, id est magna quantitate panni albi modo rotundo involutam : Georgiani autem portant melmam nigram ${ }^{13}$.

De nouveau, le vêtement est utilisé comme un marqueur identitaire ethnique et indique une façon de s'habiller commune aux orientaux, qu'ils soient chrétiens ou musulmans.

Malgré tout, les vêtements des chrétiens orientaux paraissent dans l'ensemble peu retenir l'attention des pèlerins et des chroniqueurs, sauf lorsque ceux-ci, comme dans les exemples cités, les mentionnent au service d'une argumentation plus générale, ou comme un élément parmi d'autres

\footnotetext{
13 «Les Géorgiens sont vêtus à la manière des Sarrasins, excepté la tête. Les Sarrasins portent en effet une melma blanche, c'est-à-dire une longueur importante d'étoffe blanche, enroulée en rond, tandis que les Géorgiens portent une melma noire»" (JaCQues de Vérone, Liber Peregrinationis, Ugo Monneret DE ViLlard et Giuseppe TUCCI (éd.), Rome, « Il Nuovo Ramusio », 1950, p. 34. Nous traduisons).
} 
de la caractérisation ethnique. L'explication réside sans doute dans le fait qu'ils ne trouvent rien d'extraordinaire dans les vêtements civils portés par les laïcs, et que les vêtements monastiques ou cléricaux ne diffèrent pas suffisamment de ce qu'ils connaissent en Occident pour qu'ils prennent la peine de les mentionner ${ }^{14}$.

En revanche, les vêtements des musulmans, et surtout ceux portés par les femmes et qui les cachent entièrement au regard, sont presque systématiquement relevés et commentés par les auteurs de récits de pèlerinage, dans une perspective plus orientale et exotique. Cependant, la description des vêtements portés par les femmes musulmanes devient rapidement un topos. Leurs vêtements ne sont donc pas évoqués comme une nouveauté par chaque auteur, mais sont sans doute attendus par leurs lecteurs comme un élément du décor oriental, dans la mesure où cette coutume diffère de ce qu'ils peuvent observer dans leur environnement familier et invite à tous les fantasmes sur la beauté voilée des orientales. Dans une perspective similaire, deux éléments du vêtement des chrétiens orientaux font exception et retiennent l'attention de nombreux pèlerins : le turban, commun aux Sarrasins et aux chrétiens d'Orient, qui répond donc au critère de la nouveauté et de l'orientalisme, et la ceinture, dont le port est imposé aux chrétiens d'Orient par les Sarrasins.

\footnotetext{
${ }^{14}$ Burchard précise au sujet des Arméniens : «Induuntur autem sacerdotes eorum et epicospi, sicut et nostri » («Leurs prêtres et leurs évêques sont habillés comme les nôtres ", BURChard DE MONT SiOn, Descriptio Terrce Sanctoe, dans Peregrinatores Medii Aevi quatuor..., éd. cit., p. 92. Nous traduisons). Sur les vêtements ecclésiastiques des Nubiens, des coptes, des Syriens et des Arméniens, voir notamment Karel C. INNEMÉE, Ecclesiastical dress in the medieval Near East, Leiden/New York/Köln, Brill, « Studies in Textile and Costume History », 1992.
} 


\section{Turbans et ceintures, des signes discriminants sous la souveraineté musulmane}

Burchard de Mont Sion est le premier pèlerin à signaler le port, par les Syriens, d'une ceinture qui sert à les distinguer des musulmans. Au $\mathrm{XIV}^{\mathrm{e}}$ siècle, tous les auteurs de récits de pèlerinage qui s'intéressent aux chrétiens d'Orient évoquent, parmi les nations orientales, les « chrétiens de la ceinture ». Comme Burchard, ils les rencontrent en Syrie, et notamment à Jérusalem, mais aussi, de plus en plus, en Égypte. En effet, à plusieurs reprises les conquérants musulmans imposent le port de « la ceinture » aux dhimmis, c'est-à-dire aux chrétiens autochtones et aux juifs. L'origine des mesures imposant le port de signes distinctifs remonterait au calife 'Umar ibn al-Khattāb (634-644). Entre autres prescriptions, les dhimmis auraient accepté de se serrer la taille au moyen du zunnār, c'est-à-dire d'une ceinture spéciale, portée par eux seuls ${ }^{15}$. Elle signifie simplement que ceux qui la ceignent appartiennent aux religions du Livre sans être musulmans. L'identification précise des chrétiens pose donc problème pour les Occidentaux. Lors de sa description de l'Égypte et des habitants qui la peuplent, le franciscain irlandais Symon Semeonis, qui se rend en Terre sainte en 1323, rend compte de cette difficulté :

Inhabitantur enim Saracenis, Christianis, Grecis, Scimaticis, [et] Judeis perfidis, qui omnes in habitu et modo incedendi conveniunt, exceptis Chritianis qui Frangi nuncupantur, de quibus dictum est. Et in nullo quoad habitum distinguuntur, nisi penes colorem panni, quem sibi ad capud multipliciter ligant, et zonam sive cingulum, quo cinguntur Christiani de cinctura, qui sunt Greci et Jacobite, de quibus dictum est [...] Qui [Saraceni] caputio non utuntur, sed loco eius sibi curiose circa caput pannum album lineum vel bumbacinum imponentes ligant, collum in nullo cooperientes. Judei vero

\footnotetext{
${ }^{15}$ Sur la convention de 'Umar d'après Turtūšs̄ voir Antoine FATTAL, Le Statut légal des non-musulmans en pays d'Islam, Beyrouth, Institut de Lettres orientales de Beyrouth, Imprimerie catholique, « Recherches », t. X, 1958, p. 60-69.
} 
qui sunt Rabanym pannum glaucum sive coccinum consimiliter ligant [et] portant, quo distinguuntur et cognoscuntur, et dicti Christiani, videlicet alii a Frangis, blavii seu rubei coloris, et cingulo exterius cinguntur de serico facto vel lino, a quo de cinctura nuncupantur ${ }^{16}$.

Symon identifie donc ces chrétiens aux Grecs - c'est-à-dire probablement aux melkites -, puis aux jacobites - c'est-à-dire sans doute aux coptes, qui sont monophysites comme ces derniers ${ }^{17}$. Il explique l'origine et la signification de l'expression qui les désigne par le port d'une ceinture (cingulum). Il ajoute à celle-ci un autre signe distinctif, le turban, dont la couleur est imposée et qui distingue les dhimmis des musulmans, et les dhimmis entre eux. Jacques de Vérone mentionne également les deux vêtements, le turban et la ceinture, et fait dépendre le nom des « chrétiens de la ceinture », qu'il identifie par ailleurs comme des jacobites, de cette dernière $^{18}$. Comme Symon, la plupart des pèlerins qui mentionnent les

${ }^{16}$ « En effet dans cette ville habitent des Sarrasins, des Grecs, des schismatiques [et] des juifs perfides qui concordent tous dans leur comportement et dans leur manière de marcher, à l'exception des chrétiens qui sont appelés Francs, dont il a déjà été question. Rien ne les distingue dans leur apparence, si ce n'est la couleur du morceau d'étoffe dont ils entourent plusieurs fois leur tête, et la bordure du vêtement ou la ceinture dont les chrétiens de la ceinture, qui sont grecs ou jacobites, se ceignent et tirent leur nom [...] Les Sarrasins n'utilisent pas le capuchon, mais à la place, ils lient, en la plaçant curieusement [avec soin] autour de leur tête, une étoffe blanche en lin ou en coton [en soie ?], mais sans recouvrir leur cou. Les juifs qui sont rabbins lient et portent semblablement une étoffe verdâtre ou écarlate, grâce à laquelle ils sont distingués et reconnus, et lesdits chrétiens, c'est-à-dire les autres chrétiens que les Francs, [portent une étoffe] de couleur bleue ou rouge et se ceignent, par-dessus leurs vêtements, d'une ceinture faite en soie ou en lin, d'après laquelle ils sont appelés de la ceinture » (SYMON Semeonis, Itinerarium Symonis Semeonis ab Hybernia ad Terram Sanctam, Mario EsPosito (éd.), Dublin, The Dublin Institute for Advanced Studies, « Scriptores Latini Hiberniae », 1960, chapitre 34, p. 58. Nous traduisons).

17 Anna-Dorothee von den Brincken avance l'idée que, dans le contexte égyptien, il s'agit le plus souvent de chrétiens melkites. Elle se fonde sur la meilleure intégration des coptes à la communauté musulmane égyptienne pour expliquer que cette mesure discriminante les touche moins (Anna-Dorothee VON DEN BRINCKEN, Die "Nationes christianorum orientalium" im Verständnis der lateinischen Historiographie : von der Mitte des 12.bis in die zweite Hälfte des 14. Jahrhunderts, Cologne, Böhler, « Kölner historische Abhandlungen », 22, 1973, p. 81).

18 «In Kayro et Babilonia. sunt multi cristiani. qui vocantur cristiani de centura. eo quod cincti vadunt ad differenciam Saracenorum, qui non portant cingulum : sed portant 
chrétiens de la ceinture associent leur nom au port d'une ceinture ou d'un turban et en précisent la couleur ${ }^{19}$. Toutefois Jean de Mandeville ne mentionne que la ceinture qu'ils portent tandis que Nicolas de Martoni, Simone Sigoli et Ogier d'Anglure ne mentionnent que leur turban. Le vêtement est à nouveau un critère d'identification ethnique : il permet aux latins d'appréhender un rapprochement entre les chrétiens orientaux et les Sarrasins.

in capite melmam nigram, Saraceni vero albam : vestiti autem sunt in aliis ut Saraceni » («Au Caire et à Babylone il y a beaucoup de chrétiens qui sont appelés chrétiens de la ceinture, parce qu'ils vont ceinturés, à la différence des Sarrasins, qui ne portent pas de ceinture. Mais ils portent sur la tête une melma noire, tandis que les Sarrasins en portent une blanche, pour tout le reste leurs vêtements sont semblables aux Sarrasins"», JACQUES DE VÉRONE, Liber Peregrinationis, éd. cit., p. 85. Nous traduisons).

${ }^{19}$ En voici quelques exemples : "Quidam vocantur Jacobini qui dicuntur cristiani de centura, quia vadunt induti ad modum Saracenorum excepto quod portant in capite melmam lazuram, Saraceni autem albam et vadunt cincti» («Certains sont appelés Jacobins [Jacobites], on les nomme chrétiens de la ceinture, parce qu'ils vont vêtus à la manière des Sarrasins, sauf qu'ils portent sur la tête une melma de couleur bleue, tandis que les Sarrasins en portent une blanche, et ils [les chrétiens de la ceinture] vont ceinturés », JACQUES DE VÉRONE, Liber peregrinationis, éd. cit., p. 59. Nous traduisons) ; «Des autres y a qe homme appelle Christiens de la ceinture pur ceo q'il sont ceintez par dessure » (JEAN DE MANDEVILLE, Le Livre des merveilles du monde, Christiane Deluz (éd.), Paris, Éditions du CNRS, "Sources d'histoire médiévale », 2000 , p. 249); « Sunt christiani de centura qui vadunt cum dictis vestimentis et portant capita ita ligata, verum facioli sunt coloris azolini » («Il y a les chrétiens de la ceinture qui vont, portant ces dits vêtements et la tête ainsi entourée, mais les bandelettes sont couleur d'azur », NiCOLAS DE MARTONI, Relation du pèlerinage à Jérusalem de Nicolas de Martoni, notaire italien (1394-1395), Léon LE GRAND (éd.), Paris, Ernest Leroux, extrait de la Revue de l'Orient latin, 3, 1895, p. 23.Nous traduisons); "Conosconsi queste generazioni per questo modo : I Saracini portano in capo le bende bianche, e i Guidei le bende gialle, e i Cristiani di cintura le bende azzurre, e i Samaritani le bende rosse » ("Chaque peuple est reconnaissable de cette façon. Les Sarrasins portent sur la tête un bandeau blanc, les juifs un bandeau jaune, les chrétiens de la ceinture un bandeau bleu et les Samaritains un bandeau rouge ", SimONE Sigoli, Viaggio al Monte Sinai, dans Pellegrini scrittori. Viaggiatori toscani del Trecento in Terrasanta, Antonio Lanza et Marcellina Troncarelli (éd.), Florence, Ponte alle Grazie, " Grandi opere. Testi e documenti», 1990, p. 222. Nous traduisons). Guillaume de Boldensele ne mentionne les «chrétiens de la ceinture » que dans le cadre d'une liste énumérant les différentes communautés orientales (GUILLAUME DE BOLDENSELE, Liber de quibusdam ultramarinis partibus et praecipue de Terra Sancta [ex. ronéotypés ], Christiane DeluZ (éd.), Thèse de doctorat, Paris, Sorbonne, 1972, p. 255). 
Mais la frontière entre identité ethnique et identité religieuse est alors mince. Les deux types d'identité ne sont pas clairement distingués et participent d'une commune identification de ces groupes. Si Burchard distinguait nettement le christianisme des Syriens de leur comportement et de leur apparence, ces deux derniers critères les rapprochant des Sarrasins, les pèlerins du XIV ${ }^{\mathrm{e}}$ siècle qui évoquent les chrétiens de la ceinture mêlent les deux identités - ethnique et religieuse - comme en témoigne l'emploi même de l'expression «chrétiens de la ceinture », où « chrétiens » renvoie à celle-ci et «de la ceinture» à celle-là. Il est toutefois possible de discerner deux éléments d'identification distincts. Le port du turban permet de définir un groupe qui réunit l'ensemble des habitants de Syrie ou d'Égypte, quelle que soit leur confession. Il participe alors à la définition d'une identité orientale. Quant aux couleurs de ces turbans, elles permettent de distinguer les appartenances religieuses de ceux qui les portent. Les Latins donnent des indications de couleur qui varient d'un auteur à l'autre : Symon Semeonis, Jacques de Vérone, Nicolas de Martoni et Ogier d'Anglure s'accordent sur le fait que les Sarrasins portent un turban blanc. Selon Symon Semeonis, les juifs portent un turban verdâtre ou écarlate, ce qui rejoint partiellement les observations de Nicolas de Martoni, Simone Sigoli et Ogier d'Anglure à la fin du XIV ${ }^{\mathrm{e}}$ siècle : un turban jaune pour les juifs et rouge pour les Samaritains. Chez tous, les chrétiens portent un turban bleu, mais Symon Semeonis précise qu'il peut être également rouge, et Jacques de Vérone, dans un autre passage, décrit les chrétiens comme portant un turban noir.

Ces variations s'expliquent par le fait que les prescriptions musulmanes à ce sujet ont varié selon les périodes et selon les régions ${ }^{20}$. Toutefois, la couleur blanche semble s'imposer pour les musulmans et leur

\footnotetext{
${ }^{20}$ Voir à ce sujet Antoine FATTAL, Le statut légal des non musulmans, op. cit., p. 96-110.
} 
est réservée dans plusieurs édits, le Prophète ayant précisé que Dieu appréciait les vêtements de cette couleur. Cela corrobore les observations de Symon Semeonis et Jacques de Vérone. En revanche la mention par Jacques de Vérone de turbans noirs pour les chrétiens est plus curieuse. Le noir est en effet, à l'instar du blanc, plutôt l'apanage des musulmans, en souvenir du jour de la prise de La Mecque où Mahomet portait des vêtements et un turban de cette couleur. Cependant, plusieurs documents musulmans font état du scandale du port d'un turban noir par des chrétiens et de la nécessité d'y remédier. Les chrétiens mentionnés par Jacques de Vérone sont peut-être dans ce cas et dérogent alors à la règle ${ }^{21}$. Il est toutefois le seul à faire cette remarque, dans un contexte où les autorités égyptiennes sont particulièrement méfiantes vis-à-vis des chrétiens, quoique cette méfiance vise plutôt les Francs que les chrétiens orientaux. En outre, l'historien égyptien al-Maqrīzī rapporte qu'en 1301, alors que les chrétiens et les juifs du Caire vivaient luxueusement, un vizir en provenance du Maghreb, heurté par la morgue de ces chrétiens et leur mépris des musulmans, exige et obtient le rétablissement des mesures discriminatoires, dont le port d'un turban bleu par les chrétiens, d'un turban jaune par les juifs et d'un turban rouge par les Samaritains. En 1321, soit deux ans avant la venue au Caire de Symon Semeonis et quatorze ans avant celle de Jacques de Vérone, des émeutes éclatent qui conduisent le sultan al-Nāsir à publier un nouvel édit visant à remettre en vigueur les anciennes mesures discriminatoires. Il réserve notamment la couleur bleue aux chrétiens et la couleur jaune aux juifs. Il est donc également possible que Jacques de Vérone ait commis une erreur et qu'il ait pris du bleu marine

\footnotetext{
${ }^{21}$ Il s'agit notamment de documents abbassides, le noir étant la couleur des Abbassides. Ces documents sont certes nettement antérieurs à la période à laquelle écrit Jacques de Vérone. Voir Antoine FATTAL, ibid., p. 101-102. Le califat du fatimide al-Hākim fut le seul moment où les chrétiens furent autorisés à porter un turban noir, sans doute par dérision. Mais ce n'est évidemment plus le cas dans l'Égypte du XIV ${ }^{\mathrm{e}}$ siècle.
} 
pour du noir ou bien pris un musulman pour un chrétien sous le turban de couleur noire. En revanche les couleurs les plus fréquemment citées par les pèlerins, le bleu pour les chrétiens et le jaune et le rouge pour les juifs et les Samaritains, concordent avec les édits promulgués par les autorités égyptiennes tout au long du XIV ${ }^{\mathrm{e}}$ siècle $^{22}$.

Ces deux critères - le turban et la couleur du turban - recoupent deux points de vue, même s'ils ne sont que rarement explicitement distingués par les pèlerins et les chroniqueurs : le point de vue des Latins qui s'étonnent du port du turban et ne trouvent d'ailleurs aucun terme, en latin ou en langue vernaculaire, pour désigner cette sorte de couvre-chef, et le point de vue des conquérants musulmans qui font porter des turbans de différentes couleurs aux juifs et aux chrétiens, afin de les discriminer et de s'en distinguer.

Ogier d'Anglure, noble français qui part en pèlerinage pour la Terre sainte en 1395, évoque plus longuement les «chrétiens de la ceinture » dans trois lieux différents et selon d'autres critères que le seul port d'une ceinture ou d'un turban. À Jérusalem, Ogier évoque la ségrégation urbaine entre les Sarrasins, les juifs et les «chrétiens de la ceinture $»^{23}$. Lors de sa visite du village de Bethzel, situé à proximité de Nazareth et peuplé majoritairement de «chrétiens de la ceinture», il précise qu'ils cultivent des vignes, dont il a pu apprécier le vin $^{24}$. Il s'agit ici selon toute vraisemblance de chrétiens syriens, jacobites ou melkites,

\footnotetext{
${ }^{22}$ Un troisième édit est promulgué en 1354 par l'émir mamelouk Taz contraignant les chrétiens à réduire la longueur de leurs turbans, les chrétiennes à porter des ceintures bleues et les juives des ceintures jaunes (ibid., p. 108-109).

23 «Et par dessus ces arcades se trouvent d'autres rues par lesquelles on circule normalement d'une maison à l'autre, j'entends les Sarrasins, pas les autres ! car les chrétiens de la ceinture et les Juifs qui habitent cette Sainte Cité possèdent certains lieux dans certaines rues où se trouve leur résidence " (OGIER D'ANGLURE, Journal de voyage à Jérusalem et en Égypte (1395-1396), dans Vers Jérusalem. Itinéraires croisés au XIV $V^{e}$ siècle, Michel TARAYRE et Nicole Chareyron (trad.), Paris, Les Belles Lettres, « La Roue à livres », 2008, p. 264-265).

${ }^{24}$ Ibid., p. 265.
} 
comme dans le récit de Burchard. Il les définit selon leur lieu d'habitation, leur rapport avec les Sarrasins et leur fonction économique, donc dans une perspective ethnique et non pas religieuse. Leur mention est purement descriptive et informative, sauf peut-être dans le second passage où Ogier précise qu'ils sont plus nombreux que les musulmans dans ce village. Il est en effet possible d'y voir la trace d'un topos des récits de pèlerinage selon laquelle les chrétiens sont plus nombreux que les musulmans. Enfin, Ogier évoque à nouveau les "chrétiens de la ceinture » à l'occasion de sa visite de Gaza. Il décrit alors, comme ses contemporains, l'association entre les différentes couleurs des foulards portés, et les différentes nations. Il se place là encore dans une perspective purement informative dénuée de tout esprit polémique ${ }^{25}$. Passé leur étonnement pour cette coiffe dont ils ne sont pas familiers, les pèlerins ne s'étonnent donc pas de l'utilisation d'un vêtement pour distinguer différents groupes, et reprennent même cet élément de vêtement pour désigner les chrétiens qui le portent. Cela est sans doute à mettre en relation avec des pratiques identiques en Occident, qui leur sont donc familières. Le canon 68 du quatrième concile du Latran (1215), impose aux juifs et aux musulmans de se distinguer des chrétiens par leur vêtement, les autorités séculières étant libres de préciser le caractère particulier de cet habit. C'est ainsi qu'en 1269, à la veille de son départ pour la huitième croisade, Saint Louis impose le port de la rouelle aux juifs du royaume.

\footnotetext{
25 «La différence à laquelle on reconnaît toutes ces classes de la population dans ce pays est la suivante : d'abord on identifie les Sarrasins à ce qu'ils portent un foulard [faissel] en tissu blanc sur la tête. Les Sarrasins arabes se reconnaissent au foulard blanc qu'ils portent sur la tête mais ils nouent toujours sous la gorge les bouts de la coiffe ou de la toile dont ce foulard est fait. Les chrétiens de la ceinture se reconnaissent à ce qu'ils portent un foulard de toile teinte de couleur bleue. Les juifs se reconnaissent au fouleur de toile teinte en jaune sur leur tête. Les Samaritains dont je viens de parler, sont identifiés à leur coiffe de linge blanc, d'une toile teinte couleur fleur de pêcher, plus claire que sanguine. Mais dans tous les habits et ornements de toutes ces populations, on ne distingue aucune autre marque de quelque façon que ce soit. Ils le portent tous de la même façon, à l'exception des différences évoquées dans ces coiffes » (ibid., p. 267).
} 


\section{L'altérité vécue : entre représentations occidentales et acculturation lexicale}

Le port de la ceinture est donc compris par la majorité des pèlerins comme un signe discriminant imposé aux dhimmis par les différents pouvoirs musulmans. Deux marchands toscans qui partent en pèlerinage en Terre sainte à la fin du $\mathrm{XIV}^{\mathrm{e}}$ siècle font exception. Lors de leur visite du Caire, Lionardo Frescobaldi et Simone Sigoli, donnent une toute autre explication à l'expression de «chrétiens de la ceinture », qui renverrait selon eux à un épisode de l'Assomption. En effet, ils associent ces « chrétiens de la ceinture » aux chrétiens convertis par Thomas, et donc aux Indiens $^{26}$. Leur confusion s'explique par l'association établie en Occident entre saint Thomas et l'Inde, via la tradition de l'apostolat indien de

\footnotetext{
${ }^{26}$ Lionardo Frescobaldi affirme en effet: «Questi di cintura converti San Tomaso, e però si chiamano di cintura, perchè Nostra Donna gli diè la cintola sua quando n'andò in Cielo » («Ceux de la ceinture ont été convertis par saint Thomas, ils s'appellent de la ceinture parce que Notre Dame lui a donné sa ceinture quand elle est montée au ciel », Lionardo di Niccolò FrescoBALDI, Pellegrini scrittori: viaggiatori toscani del Trecento in Terrasanta, A. LANZA et M. TronCARELli (éd.), Florence, Ponte alle Grazie, «Grandi opere. Testi e documenti », 1990, p. 94. Nous traduisons). Quant à Simone Sigoli, il explique: "Ora ti parti e vattene nella cità di Babilonia. E brievemente andammo a vicitare cinque chiese, che ancora sono in piede a onore di Dio e delle santa fede cristiana. La prima che noi vicitammo si è la chiesa dove personalmente istette a predicare santo Tommaso apostolo, e quivi fece molti miracoli per grazia di Dio. Sarebbe troppo lungo a scrivere tutti quelli che si convertirono per le predicazioni di santo Tommaso; e chiamansi cristiani della cintura, e questo nome è dirivato che, come voi sapete, quando la nostra Donna Vergine Maria n'ando in cielo lascio la cintura sua a santo Tommaso apostolo. I Cristiani delle cintura sono grandissima quantità di gente in molti paesi, e massimamente in India " («Nous sommes partis et avons gagné la cité de Babylone [Le Caire]. Nous avons brièvement visité cinq églises, dans lesquelles on honore encore Dieu et la sainte foi chrétienne. La première que nous avons visitée est l'église où a prêché personnellement saint Thomas l'Apôtre et où il a accompli de nombreux miracles par la grâce de Dieu. Il serait trop long de décrire tous ceux qui se sont convertis suite à la prédication de Thomas. Il y a ceux qui s'appellent les chrétiens de la ceinture et dont le nom est dérivé, comme vous savez, de la ceinture que notre Dame la Vierge Marie a donnée à saint Thomas l'Apôtre lorsqu'elle est allée au ciel. Les chrétiens de la ceinture sont un peuple très nombreux, présent dans beaucoup de pays et particulièrement en Inde », SimONE SigOLI, Viaggio al Monte Sinai, éd. cit., p. 186. Nous traduisons).
} 
saint Thomas ${ }^{27}$, et par leur commune origine toscane. La relique de la sainte Ceinture est en effet conservée et vénérée depuis le milieu du $\mathrm{XII}^{\mathrm{e}}$ siècle dans la chapelle du Duomo de Prato, en Toscane ${ }^{28}$. Il s'agit de la ceinture que la Vierge aurait donnée à saint Thomas, qui ne voulait pas croire à l'Assomption. La tradition date la translatio de la relique de l'année $1141^{29}$. La relique aurait été rapportée à Prato par un jeune marchand fourreur de la ville, Michele, au retour d'un pèlerinage à Jérusalem. Elle lui aurait été remise en dot à l'occasion de son mariage secret avec une jeune fille, prénommée Maria, dont la lignée masculine

${ }^{27}$ La tradition qui attribue à Thomas l'évangélisation de l'Inde a été répandue par les Acta Thomce, rédigés en syriaque et attribués à Bardesanes d'Édesse (154-222). Sur la tradition de l'apostolat de Thomas en Inde, voir Albrecht DiHLE, « Neues zur Thomas Tradition ", repris dans Antike und Orient. Gesammelte Aufsätze hrsg. von V. Pöschl und H. Petermann, Heidelberg, C. Winter, « Supplemente zu den Sitzungsberichten der Heidelberger Akademie der Wissenschaften, Philosophisch-Historische Klasse », 1984, p. 61-77. Pierre Schneider donne la liste des auteurs chrétiens antiques qui évoquent la mission de Thomas en Inde : Grégoire de Nazianze, Gélase de Cyzique, Paulin de Nôle, Chromace d'Aquilée, Épiphane de Constantia et Grégoire de Tours. Voir à ce sujet Pierre SCHNEIDER, L'Éthiopie et l'Inde. Interférences et confusions aux extrémités du monde antique (VIII siècle av. J.-C.-VI siècle ap. J.-C.), Rome, École française de Rome, «Collections de l'École française de Rome », 2004, p. 138. Ces actes ont inspiré la légende de saint Thomas, telle que la rapporte Jacques de Voragine (JACQUES De Voragine, La Légende dorée, Alain Boureau, Pascal Collomb, Monique Goullet, Laurence Moulinier et Stefano Mula (trad.), Paris, Gallimard, «Bibliothèque de la Pléiade », 2004, p. 41 sur l'envoi de Thomas par le Seigneur en mission en Inde et p. 44-48 sur la prédication de Thomas en Inde).

${ }^{28}$ Sur la relique de la sacra cintola de Prato, voir Anna Imelde GALLETTI, « Storie della Sacra Cintola (schede per un lavoro da fare a Prato) », dans Toscana et Terra Santa nel Medioevo, Franco CARDINI (dir.), Firenze, Alinea, coll. « Italia, Oriente, Mediterraneo, , 1982, p. 317-338. Elle y transcrit l'Hystoria Cinguli M(arie) Virg(inis). Voir également Mario RosA, "La ceinture de la Vierge à Prato. De la religion civique à la piété éclairée ", dans Reliques modernes : cultes et usages chrétiens des corps saints des Réformes aux révolutions, Philippe BOUTRY, Pierre Antoine FABRE et Dominique JULIA (dir.), 2 vol., Paris, Éditions de l'École des Hautes Études en Sciences Sociales, «En temps et lieux ", 2009, vol.1, p. 281-312. La ceinture symbolise la virginité de la Vierge, tout en constituant une confirmation de l'Assomption corporelle de Marie. Si la relique n'est pas exclusivement présente au Prato, la tradition qui relie la sainte Ceinture à saint Thomas est propre à la cité toscane. Cela explique l'association faite par les deux marchands florentins entre les chrétiens de la ceinture et les Indiens, évangélisés par l'apôtre, association que l'on ne retrouve dans aucun autre récit de pèlerinage à ma connaissance.

${ }^{29}$ Fondée sur les deux Historice citées ci-avant, l'anonyme et celle de Guizzelmi. 
était exclusivement composée de prêtres orientaux ${ }^{30}$. La légende elle-même symbolise l'idée d'une union entre l'Occident et l'Orient chrétien via la translatio d'un héritage oriental - ici religieux - en Occident, rejoignant ainsi la rhétorique de la translatio studii et imperii. La légende de la ceinture remise par la Vierge à saint Thomas lors de l'Assomption est certes popularisée en Occident par la Legenda aurea de Jacques de Voragine ${ }^{31}$. Néanmoins, cette légende n'étant rapportée par aucun autre pèlerin pour définir les chrétiens de la ceinture, l'origine toscane de Frescobaldi et Sigoli est sans doute déterminante dans l'association qu'ils font entre chrétiens de la ceinture et chrétiens de saint Thomas. Leur identification erronée est donc le résultat de la projection de leurs représentations de chrétiens toscans sur une réalité orientale qui leur est étrangère et qu'ils tentent ainsi de rendre plus familière.

Les efforts consentis par les pèlerins pour décrire le turban, ce couvre-chef particulier, dans la mesure où le vocabulaire latin ou vernaculaire à leur disposition ne leur permet pas de se référer à un terme précis et connu de tous, résulte de la même démarche, tout en témoignant de leur curiosité pour un élément de vêtement qui sort de l'ordinaire. Ils tentent alors, par le biais de leurs descriptions ou par celui des termes qu'ils emploient, de trouver des moyens pour le rendre familier à leurs lecteurs. Symon Semeonis parle de morceaux d'étoffe (panni) qu'ils s'enroulent plusieurs fois autour de la tête. Nicolas de Martoni évoque la tête entourée et les bandelettes des chrétiens qu'il rencontre chez le patriarche copte du Caire. Simone Sigoli parle de bende. Ogier d'Anglure utilise le terme faissel (foulard).

\footnotetext{
${ }^{30}$ Mario RosA, « La ceinture de la Vierge à Prato », art. cit., p. 284.

${ }^{31}$ L'origine de cette légende remonte à un apocryphe du $\mathrm{VI}^{\mathrm{e}}$ siècle, le Transitus Marice du pseudo Joseph d'Arimathie. Ce récit, connu en Occident, constitue la source de Jacques de Voragine.
} 
Quant aux pèlerins Jacques de Vérone, qui écrit en latin, et Frescobaldi, qui écrit en toscan, ils utilisent un terme identique : melma. La première fois que Jacques l'utilise, il y appose une définition - une grande quantité d'étoffe enroulée en rond - ce qui indique que le terme n'est pas habituel, et il le latinise en le déclinant ${ }^{32}$. Frescobaldi le reprend tel quel dans son récit de pèlerinage, sans toutefois le décliner, ni indiquer, par une définition, la nouveauté de ce terme : «In capo portano uno cappelletto involtovi intorno una melma bianca, attorciata alla Saracinesca, di tela di lino $»^{33}$. D'après Ugo Monneret de Villard, il s'agit de la transformation du mot arabe 'imāma, qui signifie «turban » ${ }^{34}$. Les deux auteurs utilisent donc un mot dérivé de l'arabe afin de désigner une réalité arabe. Le terme est passé de l'arabe au latin, puis du latin au toscan. Soit Frescobaldi utilise le récit de Jacques de Vérone pour rédiger son propre récit, soit il le tire du même terme arabe entendu en Égypte, soit entre le premier tiers et la fin du $\mathrm{XIV}^{\mathrm{e}}$ siècle, le terme melma est passé du latin au toscan. Dans tous les cas, la littérature de pèlerinage, qui reflète le contact direct entre deux aires géographiques et culturelles distinctes, semble être un vecteur de transformation et d'enrichissement des langues occidentales, qu'il s'agisse du latin ou des langues vernaculaires, en l'occurrence le toscan. Les termes arabes sont utilisés dans une forme latinisée mais leur extranéité linguistique est signalée, soit par des incises du type «a Sarracenis

\footnotetext{
32 «... quia Saraceni portant melmam albam, id est magna quantitate panni albi modo rotundo involutam » («... parce les Sarrasins portent une melma blanche, c'est-à-dire une grande quantité d'étoffe blanche enroulée en rond », JACQUES DE VÉRONE, Liber Peregrinationis, éd. cit, p. 33. Nous traduisons). Jacques de Vérone utilise à nouveau ce terme pour désigner le turban porté par les chrétiens de la ceinture, probablement des coptes puisqu'ils les identifient comme des jacobites, donc comme des chrétiens monophysites : «... excepto quod portant in capite melmam lazuram » (Ibid., p. 59. Pour la citation complète du passage et sa traduction, voir la note 10).

${ }^{33}$ Lionardo FrescobALdI, Pellegrini scrittori..., éd. cit., p. 78. «Sur la tête ils portent un petit chapeau, une melma blanche, entourée sur elle-même et enroulée à la sarrasine, en toile de lin » (nous traduisons).

${ }^{34}$ JACQUES DE VÉRONE, Liber Peregrinationis, éd. cit., note 68, p. 165.
} 
dicitur », soit par l'apposition d'une définition. Dans le cas de Frescobaldi, le terme melma passe de la langue arabe au toscan, sans doute via le latin, sans que son étymologie ou son origine linguistique soit spécifiées. Deux hypothèses sont possibles : soit il les ignore et se contente de le recopier de récits antérieurs, soit le terme melma est suffisamment assimilé dans le contexte vernaculaire. Il n'a alors pas jugé nécessaire de signaler son étymologie ou bien celle-ci a tout simplement été oubliée et ce terme devient un élément à part entière de la langue occidentale dans laquelle il est utilisé.

L'habit fait donc bien, sinon le moine, du moins l'Oriental! Chez les deux dominicains de la fin du XIII ${ }^{\mathrm{e}}$ siècle, Burchard de Mont Sion et Riccold de Monte Croce, les vêtements deviennent costumes et révèlent l'intériorité de ceux qui les portent, en les incluant dans un ensemble qui transcende les différences entre chrétiens d'Occident et chrétiens d'Orient pour regrouper des chrétiens unis par leur mode de vie évangélique. À l'inverse, le port de la ceinture et du turban est, à juste titre, perçu par les pèlerins latins comme un signe discriminant, et la nouveauté même du turban devient alors un élément de l'identification ethnique des chrétiens orientaux comme appartenant à un groupe distinct de celui des Latins et qui les renvoie à leur altérité orientale. En outre, la mention du port d'une ceinture ou d'un turban par les chrétiens du Moyen-Orient suit une tendance à la sécularisation du contenu des récits de pèlerinage qui commence au $\mathrm{XIV}^{\mathrm{e}}$ siècle et se caractérise par une attention plus soutenue pour les réalités quotidiennes. Les chrétiens d'Orient n'échappent pas à cette évolution du regard occidental. Certes, l'essentiel des remarques à leur sujet porte toujours sur leur identité religieuse, ecclésiologique, théologique et liturgique. Toutefois, si cela reste vrai chez les auteurs issus $\mathrm{du}$ milieu monastique, les auteurs laïcs, comme les marchands toscans 
Lionardo Frescobaldi et Simone Sigoli, concentrent leurs observations sur l'apparence de ces chrétiens et de moins en moins sur leurs croyances. Un second facteur est à prendre en compte : la langue dans laquelle est rédigé le récit de pèlerinage. Force est de constater que les récits en langue vulgaire comme ceux des pèlerins toscans précités ou du noble français Ogier d'Anglure accordent davantage d'importance aux réalités séculières ainsi qu'aux activités profanes de ces «chrétiens de la ceinture», sans doute en lien avec leur lectorat, qui dépasse le cercle traditionnel des clercs. Enfin, s'il n'est pas toujours clair que l'expression «chrétiens de la ceinture » pourrait caractériser l'ensemble des chrétiens d'Orient, puisque les auteurs latins continuent à les citer parmi les fidèles des autres Églises orientales - nestoriens, coptes, maronites ou jacobites - les auteurs de récits de pèlerinage perçoivent clairement les éléments d'habillement que sont le turban et la ceinture comme des signes politiques discriminants, visant à l'identification immédiate de ceux qui les portent par ceux qui les leur imposent. 